Automatic Motor Activation by Mere Instruction

\author{
Tom Everaert \\ Marijke Theeuwes \\ Baptist Liefooghe \\ Jan De Houwer \\ Ghent University
}

Address correspondence to:

Tom Everaert

Department of Psychology

Ghent University

Henri Dunantlaan 2

B-9000 Ghent, Belgium

TEL: ++32-9-264-91-07

FAX: ++32-9-264-64-89

E-mail: Tom.Everaert@UGent.be 


\begin{abstract}
Previous behavioral studies have shown that instructions about S-R mappings can influence task performance even when these instructions are irrelevant for the current task. In the present study, we tested whether automatic effects of S-R instructions occur because the instructed stimuli automatically activate their corresponding responses. We registered the lateralized readiness potentials (LRPs) that were evoked by the instructed stimuli while participants were performing a task for which those mappings were irrelevant. Instructed S-R mappings clearly affected task performance in electrophysiological and behavioral measures. The LRP was found to deflect in the direction of the response tendency that corresponded with the instructed S-R mapping. Early activation of the instructed response was observed, but occurred predominantly in slow trials. In contrast, response conflict evoked by instructed S-R mappings did not modulate the N2 amplitude. The results strongly suggest that, like experienced S-R mappings, instructed S$\mathrm{R}$ mappings can lead to automatic response activation, but possibly via a different route.
\end{abstract}




\section{Automatic Motor Activation by Mere Instruction}

Learning through instructions is a unique human ability that offers a quick route to changes in behavior without the necessity of actual practice. It appears to be based on the implementation of verbal instructions into procedural representations which control responding (e.g., Cohen-Kdoshay \& Meiran, 2007; Liefooghe, Wenke, De Houwer, 2012; Meiran, Coles, \& Braver, 2012; Wenke, Gaschler, \& Nattkemper, 2007). Such view is supported by research demonstrating that merely instructed Stimulus-Response (S-R) mappings, which have never been applied before, can bias responding when being irrelevant. For instance, Liefooghe et al. (2012; see also Liefooghe, De Houwer, \& Wenke, 2013) used a procedure in which participants are presented with runs of trials consisting of two tasks: the inducer task and the diagnostic task (Figure 1). Each run starts with the presentation of a novel pair of S-R mappings of the inducer task that indicates how to respond to the identity of a probe stimulus that would be presented later on (e.g., if the probe stimulus is the non-word 'ady', press left). Between the presentation of the S-R mappings and the onset of the probe, several trials of the diagnostic task are presented. Both tasks share stimuli and responses, but in the diagnostic task participants respond to the orientation rather than the identity of the stimuli (e.g., if upright press left; if italic, press right). Liefooghe et al. (2012) observed that RTs in the diagnostic task were faster when the required response in the diagnostic task corresponded to the S-R mappings of the inducer task (i.e., a congruent trial such as 'ady' presented upright, requiring a left key-press), than when it did not (i.e., an incongruent trials such as 'ady' presented in italics, requiring a right key-press). A congruency effect was thus observed that was based only on the instructions of 
the inducer task. This effect is referred to as the Instruction-Based Task-Rule Congruency Effect (IB-TRCE).

The IB-TRCE and related effects, such as the instruction-based feature-binding effect (Wenke et al., 2007, 2009), the instruction-based Simon effect (De Houwer, Beckers, Vandorpe, \& Custers, 2005) or the instruction-based Flanker compatibility effect (Cohen-Kdoshay \& Meiran, 2007, 2009; Meiran \& Cohen-Kdoshay, 2012), are considered as evidence for the hypothesis that instructed S-R mappings can be implemented into functional S-R associations and this without any practice. Once these S-R associations are established, they can trigger responses even when being irrelevant for the task at hand. In other words, the assumption is made that instructed S-R mappings can lead to Automatic Instruction-Based Response Activation (AIBRA). Although there is consensus that AIBRA underlies many instruction-based effects (see Meiran et al., 2012, for a review), there is only behavioral evidence in support of this explanation. Accordingly, alternative explanations for instruction-based effects which do not call upon AIBRA have also been put forward. For instance, Cohen-Kdoshay and Meiran (2007; 2009) noted that the effects reported by Wenke et al. $(2007,2009)$ and De Houwer et al. (2005) may reflect interference between the declarative knowledge about different sets of instructions rather than actual AIBRAs. Although Cohen-Kdoshay and Meiran $(2007,2009)$ and Liefooghe et al. $(2012,2013)$ offered additional controls to rule out such alternative explanations, until now all evidence is based on the mere existence of the behavioral effects that AIBRA is supposed to explain (e.g., IB-TRCE, instruction-based flanker effect). In the present study, we therefore looked for neurophysiological signs of AIBRA. More precisely, we investigated the EEG signature 
of the IB-TRCE and focused on an important marker of motor activation: the Laterized Readiness Potential (LRP; Gratton, Coles, Sirevaag, Eriksen, \& Donchin, 1988; Smulders \& Miller, 2013).

It is a well-replicated finding that the preparation of a manual response is accompanied by a negative potential that is maximal over the motor cortex, contralateral to the responding hand (for an overview, see Smulders \& Miller, 2013). This LRP is widely accepted as a measure of hand-specific response activation. First, its locus has been shown to lie in the motor cortex (Arezzo \& Vaughan, 1975; Eimer, 1998; Gemba \& Sasaki, 1990; Okada, Williamson, \& Kaufman, 1982; Praamstra, Stegeman, Horstink, \& Cools, 1996; Requin, 1985), suggesting that the LRP has strong ties with motor preparation and response activation. Second, the LRP is an extremely valid predictor of motor responses (Gratton et al., 1988). The onset of electromyographic activity occurs reliably when the LRP reaches a certain threshold value. Third, the LRP covaries with a wide array of movement parameters. In paradigms in which the response is precued, for instance, the size of the preparatory LRP, or foreperiod LRP, increases with the number of parameters that is specified about the response to be made (e.g. Leuthold, Sommer, \& Ulrich, 1996; Wild-Wall, Sangals, Sommer, \& Leuthold, 2003).

Importantly for the present purpose, LRPs also allow the measurement of automatic response activations (e.g. Eimer, 1995; 1998; Gratton et al., 1988). This has been demonstrated by studies that relate LRP modulations to S-R congruency effects, such as the Flanker effect (e.g. Gratton et al., 1988), the Simon effect (e.g. De Jong, Liang, \& Lauber, 1994), the SNARC effect (e.g., Gevers, Ratinckx, De Baene, \& Fias, 2006), and the task-rule congruency effect in task switching (e.g., Umebayashi \& Okita, 2010). It is commonly assumed that S-R congruency effects are driven by automatic response activations that are evoked by irrelevant stimuli, stimulus 
features, or tasks (e.g., Kornblum, Hasbroucq, \& Osman, 1990, Hommel et al., 2001). LRP studies on these different S-R congruency effects have revealed modulations of the onset time and amplitude of LRPs as a function of S-R congruency.

Compared to congruent trials, incongruent trials often show an early activation of the incorrect response before the correct response is fully activated (De Jong et al., 1994; Gratton et al., 1988). Furthermore, the onset of stimulus-locked LRPs (SLRPs) is generally later for incongruent trials than for congruent trials. Usually, no such differences are observed when analyzing the onset of the LRP in response-locked segments (LRPRs; Keus, Jenks, \& Schwarz, 2005; Masaki, Wild-Wall, Sangals, \& Sommer, 2002). These effects on LRP onset demonstrate that S-R congruency mainly affects the duration of the processes occurring before response activation (SLRP onset latency) rather than the duration of response activation and motor programming (LRPR onset latency; Leuthold et al., 1996; Smulders \& Miller, 2013).

Taken together, LRP modulations offer a set of valid markers of automatic response activation. We used LRPs to investigate if merely instructed S-R mappings, that have never been applied before can indeed lead to automatic response activations. Such finding has farstretching consequences for theorization on the implementation of instructions, as it tackles the core of many accounts on instruction implementation, namely that instructions in itself can lead to AIBRA. Accordingly, the present study could provide unique evidence for the hypothesis that instructed S-R mappings can be translated into functional S-R associations even in the absence of any practice. In contrast, failing to find an effect of instruction-based task-rule congruency on the LRP signal would seriously challenge the current consensus in research on instructions, 
because this would suggest that instruction-based effects have to be explained without calling upon AIBRA.

In order to investigate the hypothesis that merely instructed task-irrelevant S-R mappings can lead to AIBRA, EEGs were measured in the procedure developed by Liefooghe et al. $(2012,2013)$. We hypothesized that if the mere instruction of S-R mappings of the inducer task leads to AIBRA in the diagnostic task, then the instructed S-R mappings would lead to larger LRPs on congruent trials compared to incongruent trials. Furthermore, the LRPs of incongruent trials are predicted to display an early activation of the incorrect response before the correct response is fully activated. Congruent trials are also thought to evoke LRPs with an earlier onset than incongruent trials in the stimulus-locked segments, as S-R congruency mainly affects the duration of the processes occurring before the onset of the stimulus-locked LRP (Leuthold et al., 1996; Smulders \& Miller, 2013).

In addition, S-R congruence is known to evoke a distinct modulation of the EEG signal that could be of interest to the current study. Incongruent trials often evoke a more pronounced N2 than congruent trials. This modulation, peaking approximately $300 \mathrm{~ms}$ after stimulus onset at fronto-central sites, is thought to reflect the detection of S-R conflict and likely originates from the anterior cingulate cortex (ACC; Folstein \& Van Petten, 2008; Van Veen \& Carter, 2002). We therefore investigated whether instruction-based S-R conflict also evokes a larger N2.

\section{Method}

\section{Participants}


Twenty right-handed students at Ghent University (Mage $=23, \mathrm{SD}=6,17$ women) with normal or corrected-to-normal vision signed up for the experiment on their own initiative and without any restriction on the basis of participants' sex (e.g., an equal number of men and women). They were paid $€ 20$ to participate in the study. The higher proportion of women in our sample reflects the higher proportion of women students at Ghent University, especially in the Faculty of Psychology and Educational Sciences where the experiment was run.

\section{Stimuli and Materials}

A list of 126 three-letter non-words was generated randomly using Wordgen (Duyck, Desmet, Verbeke, \& Brysbaert, 2004). For each participant, a set of 63 pairs of non-words was randomly constructed on the basis of this list. These pairs were randomly assigned to 9 blocks, each containing 7 runs. The 7 pairs of each block were randomly assigned to the 7 runs within each block ( 4 runs with 4 diagnostic trials, 2 runs with 8 diagnostic trials, and 1 run with 16 diagnostic trials.).

In each run, one of the 63 non-word pairs was used for both the inducer task and the diagnostic task. The same left- and right keys (' $a$ ' and ' $p$ ' on an AZERTY keyboard) were used for both tasks throughout the entire experiment. In the inducer task, participants responded to the identity of a probe stimulus (a non-word presented in green), on the basis of the instructed S-R mappings. In the diagnostic task, participants decided whether a non-word, presented in black was printed upright or in italic also by either pressing a left or right key. The left-right response assignment in the diagnostic task was counterbalanced across participants.

Each non-word was presented on a white background in uppercase with an Arial bold font and a size of 24 pixels. The instructed S-R mappings were presented in a similar font with a 
size of 16 pixels and randomly presented one above the other in the screen center, such that a mapping referring to a specific response key could be either on the top line or on the bottom line.

\section{Procedure}

Participants were tested individually in a dimly-lit room by means of a personal computer with an Intel Pentium 4 processor and a 17-inch color monitor running Tscope (Stevens, Lammertyn, Verbruggen, \& Vandierendonck, 2006). Instructions were presented on screen and paraphrased subsequently. Nine blocks of 7 randomly ordered runs were presented with a small break after each block. During each break, feedback was provided about the proportion of errors made on the diagnostic task and the inducer task.

Each run started with the presentation of the S-R mappings of the inducer task. These mappings remained on screen until participants pressed the spacebar or a maximum time of 20 seconds elapsed. Once participants pressed the spacebar, the first non-word stimulus of the diagnostic task was presented after a variable inter-trial interval randomly ranging from 750ms to $1250 \mathrm{~ms}$. Each non-word stimulus in the diagnostic task remained on screen until participants responded. Depending on the run length, participants performed 4, 8, or 16 trials of the diagnostic task also with a variable inter-trial interval. Varying the number of trials of the diagnostic task made the onset of the probe stimulus of the inducer task unpredictable, which encouraged participants to be constantly prepared to execute the inducer task (see Liefooghe et al., 2012, 2013). After the last trial of the diagnostic task, the non-word probe of the inducer task was presented. This probe remained on screen until participants responded. A new run with new non-words was automatically initiated after the abovementioned inter-trial interval. 
Maximum response time was 2000 ms for all responses. Incorrect responses were followed by the Dutch word 'FOUT' ('wrong') printed in red for 200 ms. The preparation of the participant and the performance of the experiment lasted approximately 30 minutes and 25 minutes, respectively.

\section{EEG acquisition and statistical analyses}

EEG recording was performed with a Biosemi Active Two System (http://www.biosemi.com), which measured EEGs through $128 \mathrm{Ag} / \mathrm{AgCl}$ electrodes that were placed on an elastic cap and positioned according to the $A B C$ positioning system. Two external electrodes were placed on the mastoids to perform an off-line reference of the EEGs to the averaged signal of these electrodes. An additional four electrodes were used to monitor vertical and horizontal eye movements. The EEG signal was sampled at $512 \mathrm{~Hz}$ and band-pass filtered offline between $0.03 \mathrm{~Hz}$ and $30 \mathrm{~Hz}$ with an additional notch filter at $50 \mathrm{~Hz}$ to reduce any remaining noise due to $A C$ interference.

Offline computations were performed with Brain Vision Analyzer 2.0 (Brain Products, $\mathrm{GmbH}$, Munich, Germany). The EEG signals were first segmented into broad 2500 ms epochs that were locked to stimulus presentation and aligned to a 200-ms baseline before stimulus onset. Eye-blink artifacts were corrected using the standard algorithm of Gratton, Coles, and Donchin (1983) and remaining artifacts were semi-automatically detected and rejected using a \pm $75 \mu \mathrm{V}$ criterion relative to the baseline. The segments were re-segmented into stimulus-locked epochs and response-locked epochs. The stimulus-locked epochs started $200 \mathrm{~ms}$ before stimulus onset and ended 2000 ms after stimulus onset. The response-locked epochs ranged from $650 \mathrm{~ms}$ before the response to $350 \mathrm{~ms}$ after the response. 
The LRPs were calculated separately for each experimental condition. The EEG signals measured in the C3 and C4 electrodes were used to compute the LRP according to the formula proposed by Coles (1989). The ERPs ipsilateral to the responding hand were subtracted from the ERPs contralateral to the responding hand. The resulting waves were subsequently averaged across hands. In the resulting LRP waves, the activation of the correct response relative to the incorrect response is indicated by the amplitude of the signal. Negative waves indicate stronger activation of the correct response compared to the incorrect response, while positive waves indicate stronger activation of the incorrect response compared to the correct response.

LRP amplitudes were assessed using mean amplitudes in intervals that contained the LRP according to visual inspection: 300 to $600 \mathrm{~ms}$ after stimulus onset for the SLRPs, and $300 \mathrm{~ms}$ before the response until the actual response for the LRPRs. Estimation of the LRP onsets was performed using 2UDF segmented regression (Mordkoff \& Gianaros, 2000; Schwarzenau, Falkenstein, Hoormann, \& Hohnsbein, 1998) combined with the jackknife method (Miller, Patterson, \& Ulrich, 1998; Smulders, 2010; Ulrich \& Miller, 2001). The LRP onset in each participant's jackknifed average wave was estimated by fitting two regression lines to the wave using the 'segmented' package in R (Muggeo, 2008). The onset of the LRP was defined as the breaking point between of the two regression lines. To reduce the impact of high-frequency noise on the onset detection, LRPs were low-pass filtered at $7 \mathrm{~Hz}$ with a $12 \mathrm{~dB}$ octave.

\section{Results}

Analyses focus only on the diagnostic task because only performance on this task informs us about AIBRA. The first block of the experiment was considered practice and not included for further analyses. Three hundred and eighty-four trials of the diagnostic task were 
thus retained (192 congruent and 192 incongruent trials). Only correct trials with RTs not deviating more than 2.5 standard deviation from the cell mean (Ratcliff, 1993) were considered for the analysis. This resulted in the removal of $2.8 \%$ of the trials. The artifact rejection on the EEG data led to a further removal of $12.6 \%$ of the trials.

\section{Behavioral results}

A significant IB-TRCE was observed both in the RTs, $t(19)=-3.49, p=.002, d=0.78$, and in the error percentages, $t(19)=-3.70, p=.002, d=0.83$. Participants were $17 \mathrm{~ms}(S D=21 \mathrm{~ms})$ faster in congruent trials $(M=646 \mathrm{~ms}, S D=95 \mathrm{~ms})$ compared to incongruent trials $(M=663 \mathrm{~ms}$, $S D=90 \mathrm{~ms})$. Similarly, participants made fewer errors in congruent trials $(M=3.2 \%, S D=1.9 \%)$ than in incongruent trials $(M=5.4 \%, S D=3.0 \%)$.

\section{ERP results}

LRP analysis. A clear, significant, LRP emerged for all conditions in both stimulus-locked and response-locked epochs, all $t(19)^{\prime} s>2.59$, all $p^{\prime} s<.018$ (see Figure 2 and Figure 3). Importantly, the mean amplitude of the LRPs for congruent trials was significantly larger than the mean amplitude of the LRPs for incongruent trials in the stimulus-locked LRPs, $t(19)=2.52$, $p=.021, d=0.56$, and almost significant in the response-locked LRPs, $t(19)=2.00, p=.060, d=$ 0.45 .

The LRP waves also displayed a deflection that is commonly observed in compatibility tasks (e.g. De Jong et al. 1994; Eimer, 1995; Gratton et al., 1988). The stimulus-locked LRP of the incongruent trials was characterized by a small, positive, deflection starting 150 ms after stimulus onset and lasting until 300 ms after stimulus onset. After this initial deflection the LRP shifted toward the correct response. Similarly, response-locked LRPs showed the same dip in 
activation ranging from approximately $450 \mathrm{~ms}$ to $300 \mathrm{~ms}$ before the response was given. This deflection suggests a slight activation of the incorrect response on the incongruent trials. We assessed the significance of these dips by segmenting the signal around them into 650 -ms bins, calculating the mean amplitudes of these bins, and statistically testing them against zero.

The visually present activation dip in the incompatible SLRP did not reach significance, although a slight tendency towards a positive dip was present in the $200 \mathrm{~ms}$ to $250 \mathrm{~ms}$ bin, $t(19)$ $=1.60, p=0.13, d=0.36$. In contrast, the activation dip did reach significance in the incompatible LRPR in the $-400 \mathrm{~ms}$ to $-350-\mathrm{ms}$ bin, $t(19)=2.42, p=0.03, d=0.54$. This difference in significance might be due to the fact that there is considerable variation in RTs. Averaging across trials in which the LRPs did not overlap sufficiently could have made this early activation dip less apparent in the stimulus-locked segments (e.g., Luck, 2005).

To further elucidate these results, we analyzed trials with fast and slow RTs separately by performing a median split on the data. We assumed these data could clarify the earlier results for two reasons. First, effects of S-R congruency might be more pronounced in slow trials because the likelihood that an incorrect response is activated is known to be larger in these trials (e.g. Eder, Leuthold, Rothermund, \& Schweinberger, 2012). Second, analyzing fast and slow trials separately reduces the variability in the LRPs amongst the trials, thus leading to clearer LRPs when averaging fast and slow trials (e.g. Luck, 2005; Poli, Cinel, Citi, \& Sepulveda, 2010).

In accordance with this line of thought, significant activation dips were found in SLRPs (Figure 4) and LRPRs (Figure 5) for slow trials only, $t(19)=2.46, p=.024, d=0.55$, in the $200 \mathrm{~ms}$ to $250 \mathrm{~ms}$ bin for the SLRPs, and $t(19)=2.14, p=.045, d=0.48$, in the $-400 \mathrm{~ms}$ to $-350 \mathrm{~ms}$ bin for 
the LRPRs. In the fast trials, only (at best) marginally significant activation dips were found, $t(19)$ $=1.51, p=0.15, d=0.34$, for the SLRPs, and $t(19)=1.66, p=0.11, d=0.37$, for the LRPRs. Interestingly, fast congruent trials displayed an early pre-activation of the correct hand in the 50 $\mathrm{ms}$ to $100 \mathrm{~ms}$ bin in the SLRPs, $t(19)=2.39, p=.027, d=0.54$, that reached marginal significance in the next 2 bins, $t(19)=1.81, p=.09, d=0.40$, and $t(19)=2.03, p=.06, d=0.45$, for the 100 $\mathrm{ms}$ to $150 \mathrm{~ms}$ bin and the $150 \mathrm{~ms}$ to $200 \mathrm{~ms}$ bin, respectively. The actual LRP peak started reaching significance only in the $250 \mathrm{~ms}$ to $300 \mathrm{~ms}$ bin, $t(19)=2.98, p=.008, d=0.67$. A marginal correct-hand pre-activation was found in the fast, congruent LRPRs in the -450 ms to $400 \mathrm{~ms} \operatorname{bin}, t(19)=1.69, p=.11, d=0.38$.

The segmented regression analysis on the jackknifed averages yielded clear LRP onset estimates in SLRPs and LRPRs (see Figure 2 and 3 for the regression lines). Although the SLRP onset in the congruent trials $(M=256 \mathrm{~ms})$ was earlier than the SLRP onset in the incongruent trials $(M=284 \mathrm{~ms})$, the $28-\mathrm{ms}$ difference did not reach significance, $t(19)<1$. Similarly, the earlier LRPR onset for congruent trials $(M=-276)$ compared to incongruent trials $(M=-240 \mathrm{~ms})$ was not significant, $t(19)=1.47, p=.16, d=.33$.

\section{Additional analyses}

Visual inspection of the data revealed a clear N2 (Figure 6) that peaked between $250 \mathrm{~ms}$ and 350 ms after stimulus onset and was maximal at frontal sites (Fz and its surroundings). Analyses of the mean amplitude of this peak in the aforementioned time interval on electrode Fz and the 6 surrounding more posterior electrodes did not reveal any significant differences in N2 amplitude, $t(19)<1$. No other effects emerged when analyzing fast and slow trials separately, all $t(19)^{\prime} s<1$. 
S-R congruency can also lead to fluctuations in error-related negativity (ERN), a negative wave following errors that is maximal at fronto-central sites (Falkenstein, Hohnsbein, \& Hoormann, 1991; Falkenstein, Hoormann, Christ, \& Hohnsbein, 2000; Gehring, Goss, Coles, Meyer, \& Donchin, 1993). The error percentage (4.6\%) was too small for a meaningful analysis of this component, however. Only eight participants made at least 6 usable errors, which was not sufficient to elicit a significant ERN at Fz in the $100 \mathrm{~ms}$ following an error, $t(7)=1.87, p=.10$, $d=0.75$. Unsurprisingly therefore, no significant differences were observed between the ERNs following congruent trials and the ERNs following incongruent trials, $t(7)<1$.

\section{General Discussion}

The current study investigated if responses can be activated automatically as the result of instructions about S-R mappings, a hypothesis which is central in many accounts on the implementation of instructions (e.g., Cohen-Kdoshay \& Meiran, 2007, 2009; Liefooghe et al., 2012, 2013; Wenke et al. 2007, 2009). To this end, LRP modulations associated with the IB-TRCE were investigated.

A clear IB-TRCE was present on the RTs and the error rates. Importantly, lower LRP amplitudes were observed on incongruent trials compared to congruent trials suggesting that instruction-based congruency leads to differences in response activation in the motor cortex. These differences were significant mainly in the SLRPs, which suggests that the AIBRA is probably the result of visuo-motor priming evoked by the task-irrelevant stimulus attribute (e.g., Stürmer, Ouyang, Zhou, Boldt, \& Sommer, 2013). Such differences in activation that are evoked by the stimulus might become less apparent when averaging the segments aligned to the response. In line with the observed differences in LRP amplitude, the LRPs of incongruent 
trials displayed an early deflection, showing activation of the response afforded by the solelyinstructed inducer task. This deflection was present mainly in the slow trials, as the likelihood of incorrect response activation is larger in these trials.

Analyses of the LRP onsets did not reveal any significant effects of instruction-based congruency on the SLRP onset and the LRPR onset, but the estimation of the LRP onset might not have been optimal in our study. LRP onset latencies are calculated to estimate the point in time at which the activation of the correct response starts. The LRP, however, reflects the relative activation of two responses. Trials in which incorrect responses are activated can therefore obscure the onset estimation of the correct response (e.g. Stürmer et al., 2013). Since we observed clear activation of the responses in line with the irrelevant instructions, our estimates of the LRP onset might not reflect the onset of the activation of the relevant response per se.

The critical difference compared to previous research is that the congruency effect observed in the present study is based on merely instructed S-R mappings that have never been practiced or applied overtly before. In other words, our results offer strong evidence that these effects are based on AIBRA. Neuroimaging studies suggest that a frontal parietal network involved in task-rule implementation might be instrumental for the occurrence of IB-TRCEs (e.g. Hartstra, Kühn, Verguts, \& Brass, 2011; Hartstra, Waszak, \& Brass, 2012; Ruge \& Wolfensteller, 2010). In this network, the inferior frontal sulcus appears to be implicated in the formation of S$\mathrm{R}$ links, while a sizeable part of the network is responsible for the coding of responses in areas related to motor control such as the anterior IPS, premotor cortices and the pre-SMA (Hartstra et al. 2012). Our data suggest that this instruction-related activation in the fronto-parietal 
network can be sustained over time and even lead to automatic activation in the primary motor cortex during the performance of other tasks.

While the present results indicates that instruction-based congruency effects are associated with modulations in the LRP, as it is the case for execution-based S-R congruency effects, it is important to note that the EEG signatures of both effects also seem to differ. Execution-based task-rule congruency generally leads to variations in the amplitude of the N2 (e.g. Folstein \& Van Petten, 2007), while instruction-based task-rule congruency did not seem to evoke such differences. The absence of such an effect does not seem limited to our study. Brass, Wenke, Spengler, and Waszak (2009), for instance, did not observe an effect of interference from instructed S-R mappings on activity in the ACC, the likely source of the fronto-central N2 (Folstein \& Van Petten, 2008; Van Veen \& Carter, 2002). Conversely, interference from experienced S-R mappings did evoke differences in ACC activation. This contrast might be the result of the different neural pathways that are thought to be involved instruction-based and experience-based performance. While instruction-based task execution is supposedly mediated by the prefrontal cortex, experience-based task execution has been attributed to the basal ganglia (Ramamoorthy \& Verguts, 2012) and the posterior parietal cortex (Huang, Hazy, Herd, \& O’Reilly, 2013). The prefrontal route allows for fast learning of novel, instructed S-R mappings, and is the supposed source of instruction-based S-R congruency effects. In contrast, repeated task execution triggers a slow learning mechanism in the basal ganglia that eventually bypasses the prefrontal route, which dominates task execution after sufficient practice, and thus might lead to experience-based S-R congruency effects (Ramamoorthy \& Verguts, 2012). Such account is in line with the seminal proposals by Logan (1988), who suggested that novel tasks are first 
processed through a controlled algorithmic route and subsequently available for automatic retrieval.

Previous behavioral research also indicated that instructed and practiced S-R mappings are represented by different components of memory. The common hypothesis is that instructed S-R mappings are translated into a procedural representation such as a task-set (Liefooghe et al., 2012), an event-file (Wenke et al., 2007) or a plan (Meiran et al., 2012) in short-term memory. Such a representation serves the control of actions by representing functional S-R associations that are reflexively triggered when target stimuli are presented (Hommel et al., 2001; Meiran et al., 2012). Importantly, such representation is achieved only for a relatively high degree of task readiness (Liefooghe et al., 2013; Wenke et al., 2009). Whenever preparation demands are too lenient, instruction-based S-R congruency effects are absent. In contrast, execution-based S-R congruency effects, such as the task-rule congruency effect in task switching seem to be independent of task readiness (Yamaguchi \& Proctor, 2012) and based on S-R associations in long-term memory (Meiran \& Kessler, 2008). While research on instruction implementation undeniable shows that the practice or execution of $\mathrm{S}-\mathrm{R}$ mappings is not a prerequisite to obtain S-R congruence, practice may still result in a transfer from short-term S-R associations that are formed initially on the basis of instructions to S-R associations in long-term memory.

Execution-based and instruction-based congruency effects thus seem to have different underpinnings which could have led to different modulations of the N2 amplitude. Alternatively, the absence of an effect on the N2 could also be related to the size of the S-R conflict evoked by merely-instructed task-rules. The response activation evoked by irrelevant experience-based 
task-rules seems to be stronger than the response activation evoked by irrelevant instructionbased task-rules. Evidence for this difference in response strength can be found in the generally larger effect sizes found in experience-based paradigms compared to the instruction-based paradigm used in the current study. Most studies demonstrating the impact of response conflict on N2 amplitude have employed the Eriksen flanker task (Eriksen \& Eriksen, 1974), which generally yields behavioral effect sizes larger than the one obtained in our study (IB-TRCE $=17$ $\mathrm{ms}, d=0.78)$. For instance, the flanker task used in the seminal paper of Coles, Gratton, Bashore, Eriksen, and Donchin (1985) yielded a flanker congruency effect that had an absolute size of $47 \mathrm{~ms}$ and a Cohen's $d$ of 3.29. Such large effect sizes are not uncommon for the flanker paradigm (e.g. Bartholow, Pearson, Dickter, Sher, Fabiani, \& Gratton, 2005; Hazeltine, Poldrack, \& Gabrieli, 2000; Mansfield, van der Molen, Falkenstein, van Boxtel, 2013; Van Veen \& Carter, 2002; Yeung, Botvinick, \& Cohen, 2004), which might explain why modulations of N2 amplitudes are found more readily using the flanker task. In line with this reasoning, we performed a posthoc analysis on the N2 amplitudes of the trials following a congruent trial, as congruency effects are usually larger after such trials (Gratton, Coles, \& Donchin, 1992). The IB-TRCE in these trials was fairly large on behavioral measures, IB-TRCE $=34 \mathrm{~ms}, t(19)=4.20, p<0.001, d=0.94$, and manifested itself in a marginally significant effect on N2 amplitudes in the expected direction, $t(19)=1.98, p=.06, d=0.44$ (Figure 7). This additional analysis indicates that, under conditions in which the IB-TRCE is larger, significant effects on N2 amplitudes can be observed, as is the case for execution-based congruency effects, such as the flanker effect. Modulation of the fronto-central N2 component, and therefore ACC activation, may thus be more dependent on the size of the conflict rather than the source of the conflict (i.e. instructions or experience). 
To conclude, the present study demonstrates automatic motor activation on the mere basis of task-irrelevant instructions. This motor activation indicates that instructions can lead to automatic response activations, an assumption that is at the core of accounts on instruction implementation. Finally, even without considering the different processes underlying the implementation of instructions, the present study offers a very strong proof about the power seemingly irrelevant instructions have on motor behavior. 


\section{References}

Arezzo, J., \& Vaughan, H.G. (1975). Cortical potentials associated with voluntary movements in monkey. Brain Research, 88, 99-104.

Bartholow, B.D., Pearson, M.A., Dickter, C.L., Sher, K.J., Fabiani, M., \& Gratton, G. (2005).

Strategic control and medial frontal negativity: Beyond errors and response conflict. Psychophysiology, 42, 33-42.

Brass, M., Wenke, D., Spengler, S., \& Waszak, F. (2009). Neural correlates of overcoming interference from instructed and implemented stimulus-response associations. Journal of Neuroscience, 29, 1766-1772.

Cohen-Kdoshay, O., \& Meiran, N. (2007). The representation of instructions in working memory leads to autonomous response activation: Evidence from the first trials in the flanker paradigm. Quarterly Journal of Experimental Psychology, 60, 1140-1154.

Cohen-Kdoshay, O., \& Meiran, N. (2009). The representation of instructions operates like a prepared reflex: Flanker compatibility effects that are found in the first trial following S-R instructions. Experimental Psychology, 56, 128-133.

Coles, M. G. (1989). Modern mind-brain reading: psychophysiology, physiology, and cognition. Psychophysiology, 26, 251-269.

Coles, M.G.H., Gratton, G., Bashore, T.R., Eriksen, C.W., \& Donchin, E. (1985). A psychophysiological investigation of the continuous flow model of human information processing. Journal of Experimental Psychology: Human Perception and Performance, 11, 529-553. 
De Houwer, J., Beckers, T., Vandorpe, S., \& Custers, R. (2005). Further evidence for the role of mode-independent short-term associations in spatial Simon effects. Perception \& Psychophysics, 67, 659-666.

De Jong, R., Liang, C.-C., \& Lauber, E. (1994). Conditional and Unconditional Automaticity: A Dual-Process Model of Effects of Spatial Stimulus-Response Correspondence. Journal of Experimental Psychology: Human Perception and Performance, 20, 731-750.

Duyck, W., Desmet, T., Verbeke, L., \& Brysbaert, M. (2004). WordGen: A tool for word selection and non-word generation in Dutch, German, English, and French. Behavior Research Methods, Instruments \& Computers, 36, 488-499.

Eder, A.B., Leuthold, H., Rothermund, K., \& Schweinberger, S.R. (2012). Automatic response activation in sequential affective priming: an ERP study. Social, Cognitive, and Affective Neuroscience, 7, 436-445.

Eimer, M. (1995). Stimulus-response compatibility and automatic response activation: Evidence from psychophysiological studies. Journal of Experimental Psychology: Human Perception \& Performance, $21,837-854$.

Eimer, M. (1998). The lateralized readiness potential as an on-line measure of central response activation processes. Behavior Research Methods, Instruments \& Computers, 30, 146156.

Falkenstein, M., Hohnsbein, J., Hoormann, J. (1991). Effects of crossmodal divided attention on late ERP components. II. Error processing in choice reaction time tasks. Electroencephalography and Clinical Neurophysiology, 78, 447-455. 
Falkenstein, M., Hoormann, J., Christ, S., \& Hohnsbein, J. (2000). ERP components on reaction errors and their functional significance: a tutorial. Biological Psychology, 51, 87-107.

Folstein, J.R., \& Van Petten, C. (2007). Influence of cognitive control and mismatch on the N2 component of the ERP: A review. Psychophysiology, 45, 152-170.

Gehring, W.J., Goss, B., Coles, M.G.H., Meyer, D.E., \& Donchin, E. (1993). A neural system for error detection and compensation. Psychological Science, 4, 385-390.

Gemba, H., \& Sasaki, K. (1990). Potential related to no-go reaction in go no-go hand movement with discrimination between tone stimuli of different frequencies in the monkey. Brain Research, 537, 340-344.

Gevers, W., Rattinckx, E., De Baene, W., \& Fias, W. (2006). Further evidence that the SNARC effect is processed along a dual-route architecture - Evidence from the lateralized readiness potential. Experimental Psychology, 53, 58-68.

Gratton, G., Coles, M. G. H., \& Donchin, E. (1983). A new method for off-line removal of ocular artifact. Electroencephalography and Clinical Neurophysiology, 55, 468-484.

Gratton, G., Coles, M. G. H., \& Donchin, E. (1992). Optimizing the use of information: Strategic control of activation of responses. Journal of Experimental Psychology: General, 121, 480-506.

Gratton, G., Coles, M. G. H., Sirevaag, E., Eriksen, C. W. \& Donchin, E. (1988). Pre- and poststimulus activation of response channels: a psychophysiological analysis. Journal of Experimental Psychology: Human Perception and Performance,14, 331-344.

Hartstra, E., Kühn, S., Verguts, T., \& Brass, M. (2011). The implementation of verbal instructions: An fMRI study. Human Brain Mapping, 32, 1811-1824. 
Hartstra, E., Waszak, F., \& Brass, M. (2012). The implementation of verbal instructions:

Dissociating motor preparation from the formation of stimulus-response associations. Neurolmage, 63, 1143-1153.

Hazeltine, E., Poldrack, R., \& Gabrieli, J.D.E. (2000). Neural activation during response competition. Journal of Cognitive Neuroscience, 12, 118-129.

Hommel, B., Musseler, J., Aschersleben, G., \& Prinz, W. (2001). The Theory of Event Coding (TEC): A framework for perception and action planning. Behavioral and Brain Sciences, $24,849$.

Huang, T., Hazy, T.E., Herd, S.A., \& O’Reilly, R. (2013). Assembling old tricks for new tasks: A neural model of instructional learning and control. Journal of Cognitive Neuroscience, 25, 843-851.

Keus, I. M., Jenks, K. M., \& Schwarz, W. (2005). Psychophysiological evidence that the SNARC effect has its functional locus in a response selection stage. Cognitive Brain Research, 24, 48-56.

Kornblum, S., Hasbroucq, T., \& Osman, A. (1990). Dimensional overlap - Cognitive basis for stimulus-response compatibility - A model and taxonomy. Psychological Review, 97, 253-270.

Liefooghe, B., De Houwer, J., \& Wenke, D. (2013). Instruction-based response activation depends on task preparation. Psychonomic Bulletin \& Review, 20, 481-487.

Liefooghe, B., Wenke, D., \& De Houwer, J. (2012). Instruction-based task-rule congruency effects. Journal of Experimental Psychology: Learning, Memory, \& Cognition, 38, 13251335. 
Leuthold, H., Sommer, W., \& Ulrich, R. (1996). Partial advance information and response preparation: inferences from the lateralized readiness potential. Journal of Experimental Psychology: General, 125, 307-323.

Logan, G.D. (1988). Toward an instance theory of automatization. Psychological Review, 95, 492527.

Mansfield, K.L., van der Molen, M.W., Falkenstein, M., \& van Boxtel, G.J.M. (2013). Temporal dynamics of interference in Simon and Eriksen tasks considered within the context of a dual-process model. Brain and Cognition, 82, 353-363.

Masaki, H., Wild-Wall, N., Sangals, J., \& Sommer, W. (2004). The functional locus of the lateralized readiness potential. Psychophysiology, 41, 220-230.

Meiran, N., Cole, M.W., \& Braver, T.S. (2012). When planning results in loss of control: Intention-based reflexivity and working-memory. Frontiers in Human Neuroscience, 6, 104.

Meiran, N., Cohen-Kdoshay, O. (2012). Working memory load but not multitasking eliminates the prepared reflex: Further evidence from the adapted flanker paradigm. Acta Psychologica, 139, 309-313.

Meiran, N., \& Kessler, Y. (2008). The task rule congruency effect in task switching reflects activated long term memory. Journal of Experimental Psychology: Human Perception and Performance, 34, 137-157.

Miller, J., Patterson, T., \& Ulrich, R. (1998). Jackknife-based method for measuring LRP onset latency differences. Psychophysiology, 35, 99-115. 
Mordkoff, T.J., \& Gianaros, P.J. (2000). Detecting the onset of the lateralized readiness potential: A comparison of available methods and procedures. Psychophysiology, 37, 347-360.

Muggeo, V.M.R. (2008). Segmented: an R package to fit regression models with broken-line relationships. $R$ News, $8,20-25$.

Okada, Y.C., Williamson, S.J., \& Kaufman, L. (1982). Magnetic-field of the human sensorimotor cortex. International Journal of Neuroscience, 17, 33-38.

Poli, R., Cinel, C., Citi, L., \& Sepulveda, F. (2010). Reaction-time binning: A simple method for increasing the resolving power of ERP averages. Psychophysiology, 47, 467-485.

Praamstra, P., Stegeman, D. F., Horstink, M. W. I. M., \& Cools, A. R. (1996). Dipole source analysis suggests selective modulation of the supplementary motor area contribution to the readiness potential. Electroencephalography and Clinical Neurophysiology, 98, 468477.

Ramamoorthy, A. \& Verguts, T. (2012). Word and deed: A computational model of instruction following. Brain Research, 1439, 54-65.

Ratcliff, R. (1993). Methods for dealing with reaction time outliers. Psychological Bulletin, 114, 510-532.

Requin, J. (1985). Looking forward to move soon: Ante factum selective processes in motor control. In M.I. Posner \& O.S.M. Marin (Eds.), Attention and Performance X (pp. 147167). Hillsdale, NJ: Erlbaum.

Ruge, H. \& Wolfensteller, U. (2010). Rapid formation of pragmatic rule representations in the human brain during instruction-based learning. Cerebral Cortex, 20, 1656-1667. 
Schwarzenau, P., Falkenstein, M., Hoormann, J., \& Hohnsbein, J. (1998). A new method for the estimation of the onset of the lateralized readiness potential (LRP). Behavior Research Methods, Instruments, \& Computers, 30, 110-117.

Smulders, F.T. (2010). Simplifying jackknifing of ERPs and getting more out of it: Retrieving estimates of participants' latencies. Psychophysiology, 47, 387-392.

Smulders, F. T. Y., \& Miller, J.O. (2012) The Lateralized Readiness Potential. In S.J. Luck \& E.S. Kappenman. Oxford Handbook of Event-Related Potential Components. New York, Oxford University Press.

Stevens, M., Lammertyn, J., Verbruggen F., \& Vandierendonck, A. (2006). Tscope: A C library for programming cognitive experiments on the MS Windows platform. Behavior Research Methods, 38, 280-286.

Stürmer, B., Ouyang, G., Zhou, C., Boldt, A., \& Sommer, W. (2013). Separating stimulus-driven and response-related LRP components with Residue Iteration Decomposition (RIDE). Psychophysiology, 50, 70-73.

Ulrich, R., \& Miller, J. (2001). Using the jackknife-based scoring method for measuring LRP onset effects in factorial designs. Psychophysiology, 38, 816-827.

Umebayashi, K., \& Okita, T. (2010). An ERP investigation of task switching using a flanker paradigm. Brain Research, 1346, 165-173.

Van Veen, V., \& Carter, C.S. (2002). The timing of action-monitoring processes in the anterior cingulate cortex. Journal of Cognitive Neuroscience, 14, 593-602.

Wenke, D., Gaschler, R., \& Nattkemper, D. (2007). Instructions induced feature binding. Psychological Research, 71, 92-106. 
Wenke, D., Gaschler, R., Nattkemper, D., \& Frensch, P. A. (2009). Strategic influences on implementing instructions for future actions. Psychological Research, 73, 587-601.

Wild-Wall, N., Sangals, J., Sommer, W., \& Leuthold, H. (2003). Are fingers special? Evidence about movement preparation from event-related brain potentials. Psychophysiology, 40, 7-16.

Yamaguchi, M., \& Proctor, R.W. (2012). Multidimensional vector model of stimulus-response compatibility. Psychological Review, 119, 272-303.

Yeung, N., Botvinick, M.M., \& Cohen, J.D. (2004). The neural basis of error detection: Conflict monitoring and the error-related negativity. Psychological Review, 111, 931-959. 


\begin{abstract}
Author note
Tom Everaert, Marijke Theeuwes, Baptist Liefooghe, and Jan De Houwer, Department of Experimental-Clinical and Health Psychology, Ghent University, Belgium. Correspondence concerning this article should be addressed to Tom Everaert, Ghent University, Henri Dunantlaan 2, B-9000 Ghent, Belgium. Electronic mail can be sent to Tom.Everaert@UGent.be.

Preparation of this paper was supported by Grant BOF/GOA2006/001 and Grant BOF09/01M00209 of Ghent University. We would like to thank Helen Tibboel for her valuable assistance in collecting data during this study.
\end{abstract}




\section{Figure captions}

Figure 1. Outline of a run of the inducer task and the diagnostic task.

Figure 2. Grand average stimulus-locked lateralized readiness potentials as a function of compatibility. $S$ = stimulus onset. Straight lines indicate the regression lines estimated with the segmented regression procedure. The breaking-points indicate the estimated LRP onset.

Figure 3. Grand average response-locked lateralized readiness potentials as a function of compatibility. $R$ = response onset. Straight lines indicate the regression lines estimated with the segmented regression procedure. The breaking-points indicate the estimated LRP onset.

Figure 4. Grand average stimulus-locked lateralized readiness potentials as a function of compatibility and response speed. $\mathrm{S}=$ stimulus onset.

Figure 5. Grand average response-locked lateralized readiness potentials as a function of compatibility and response speed. $\mathrm{R}=$ response onset.

Figure 6. Grand average waveforms at electrodes Fz (C21) and 6 surrounding, more posterior electrodes as a function of compatibility.

Figure 7. Grand average waveforms of trials following congruent trials at electrodes Fz (C21) and 6 surrounding, more posterior electrodes as a function of compatibility. 
Figure 1

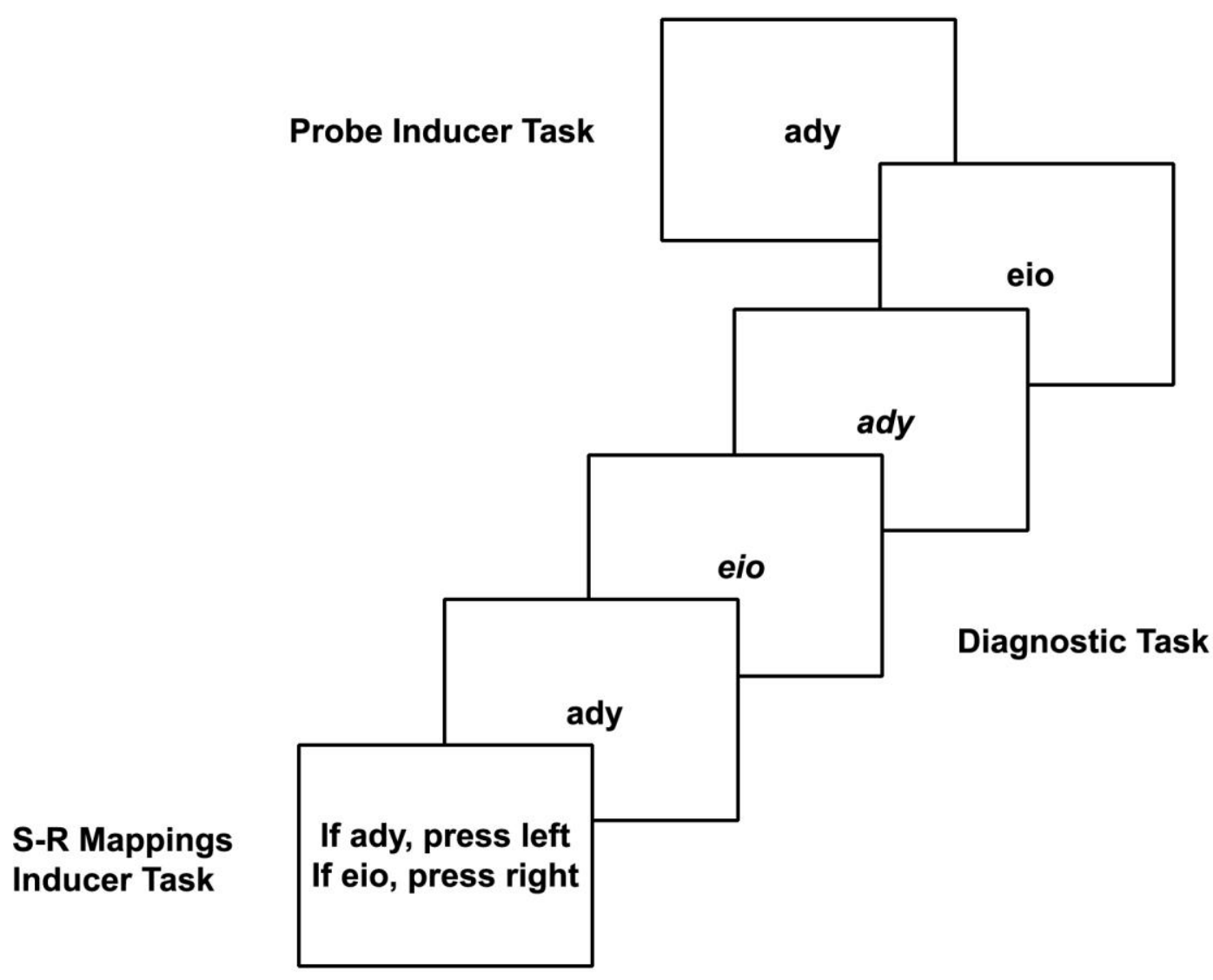


Figure 2

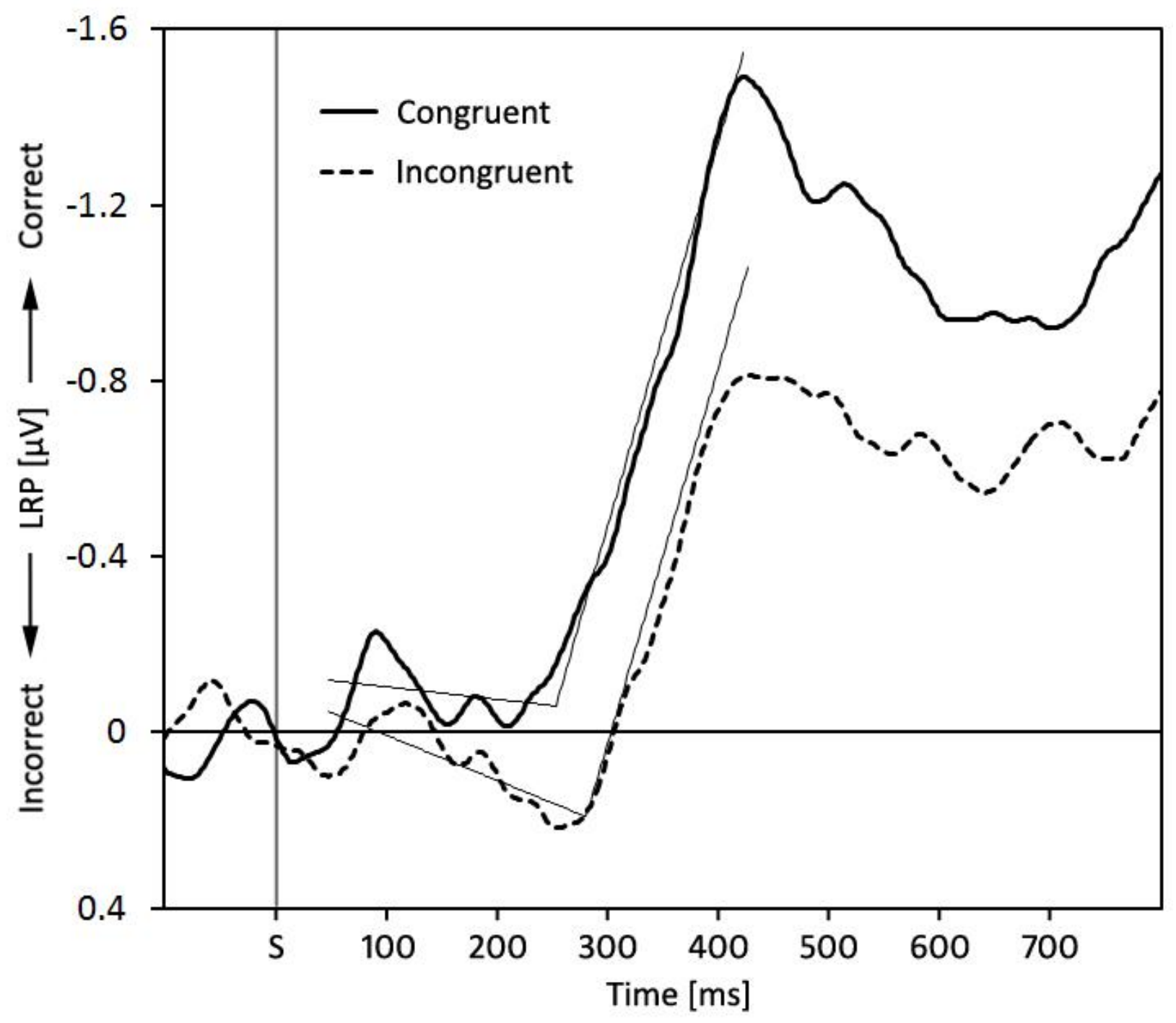


Figure 3

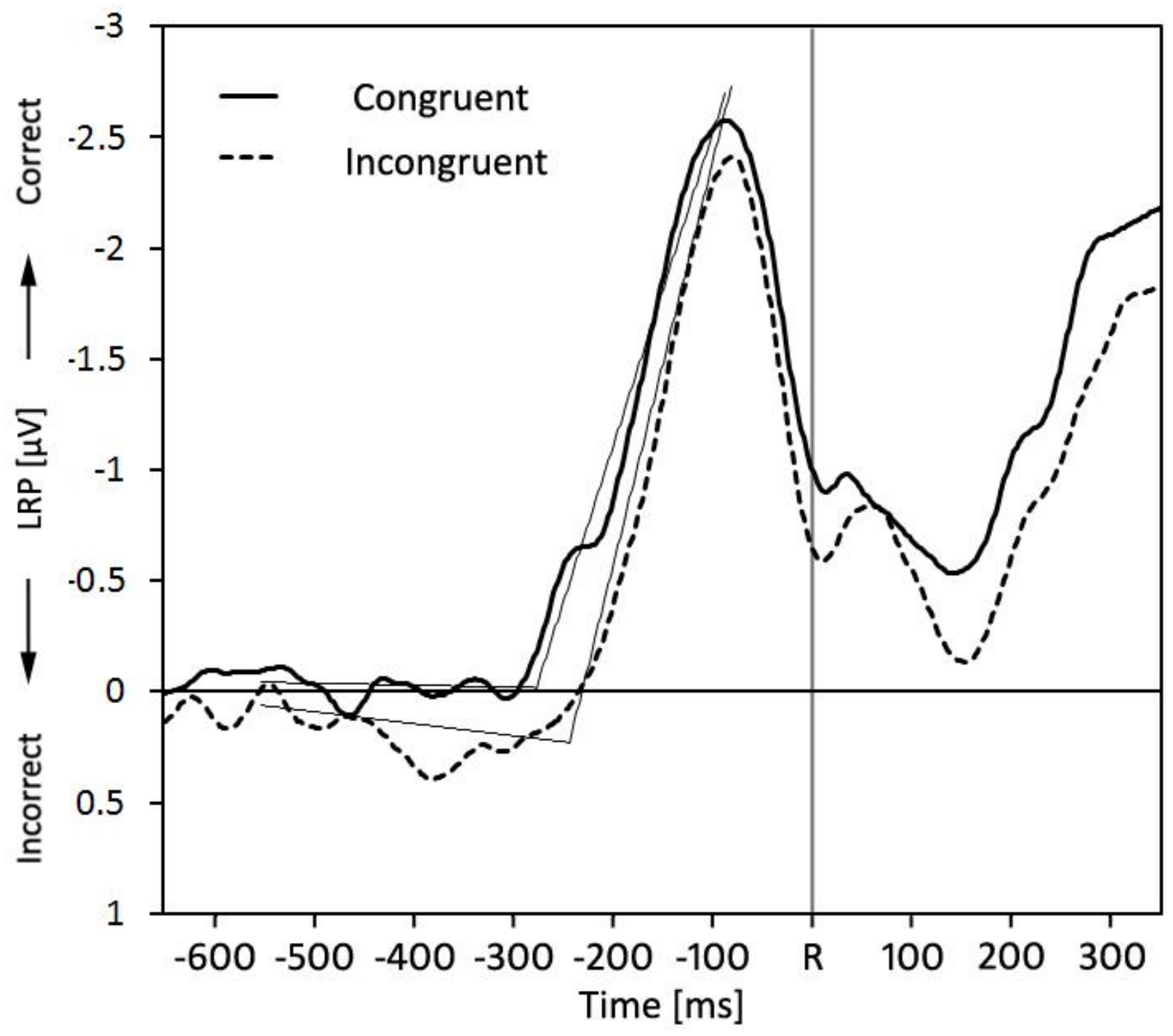


Figure 4

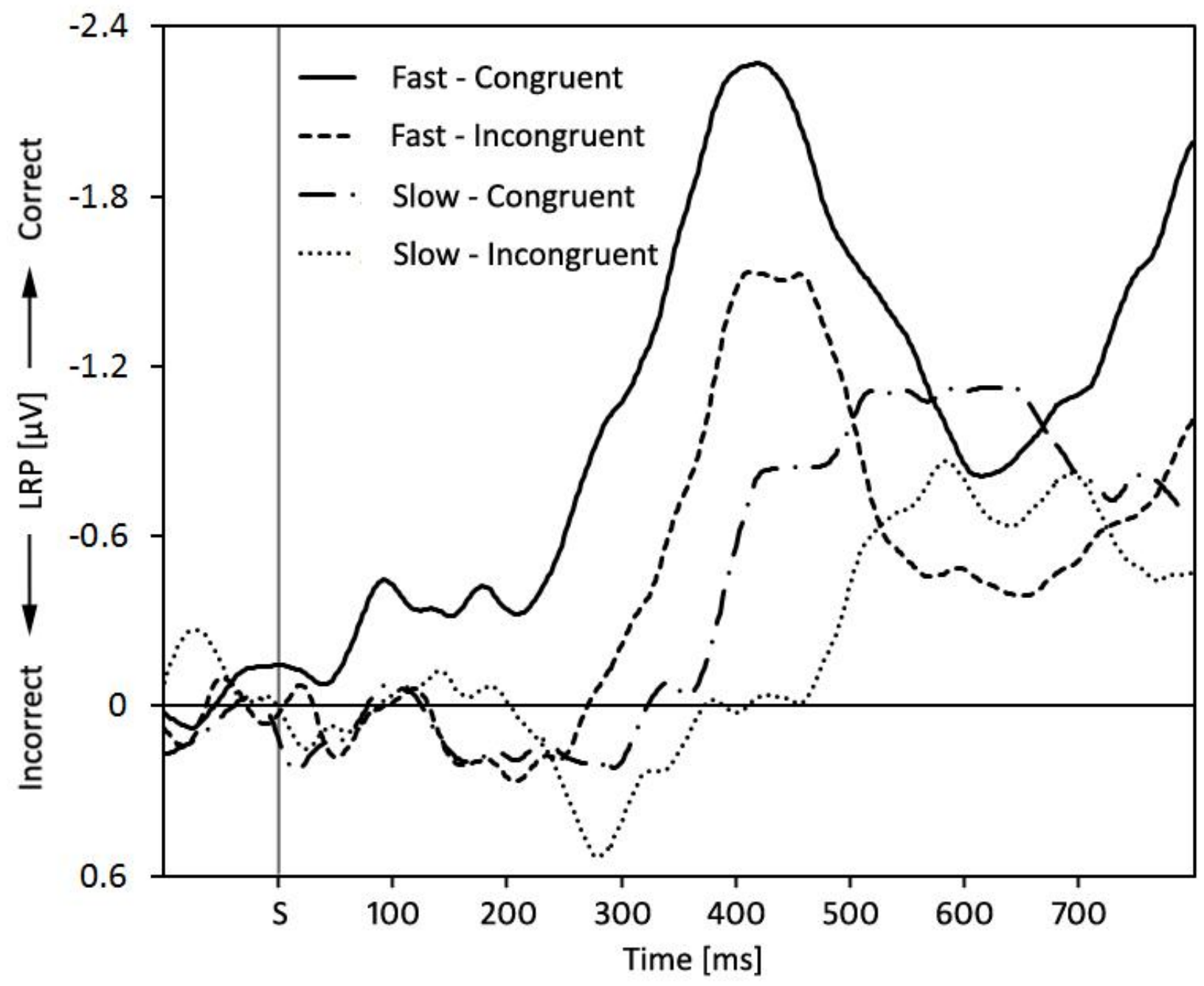


Figure 5

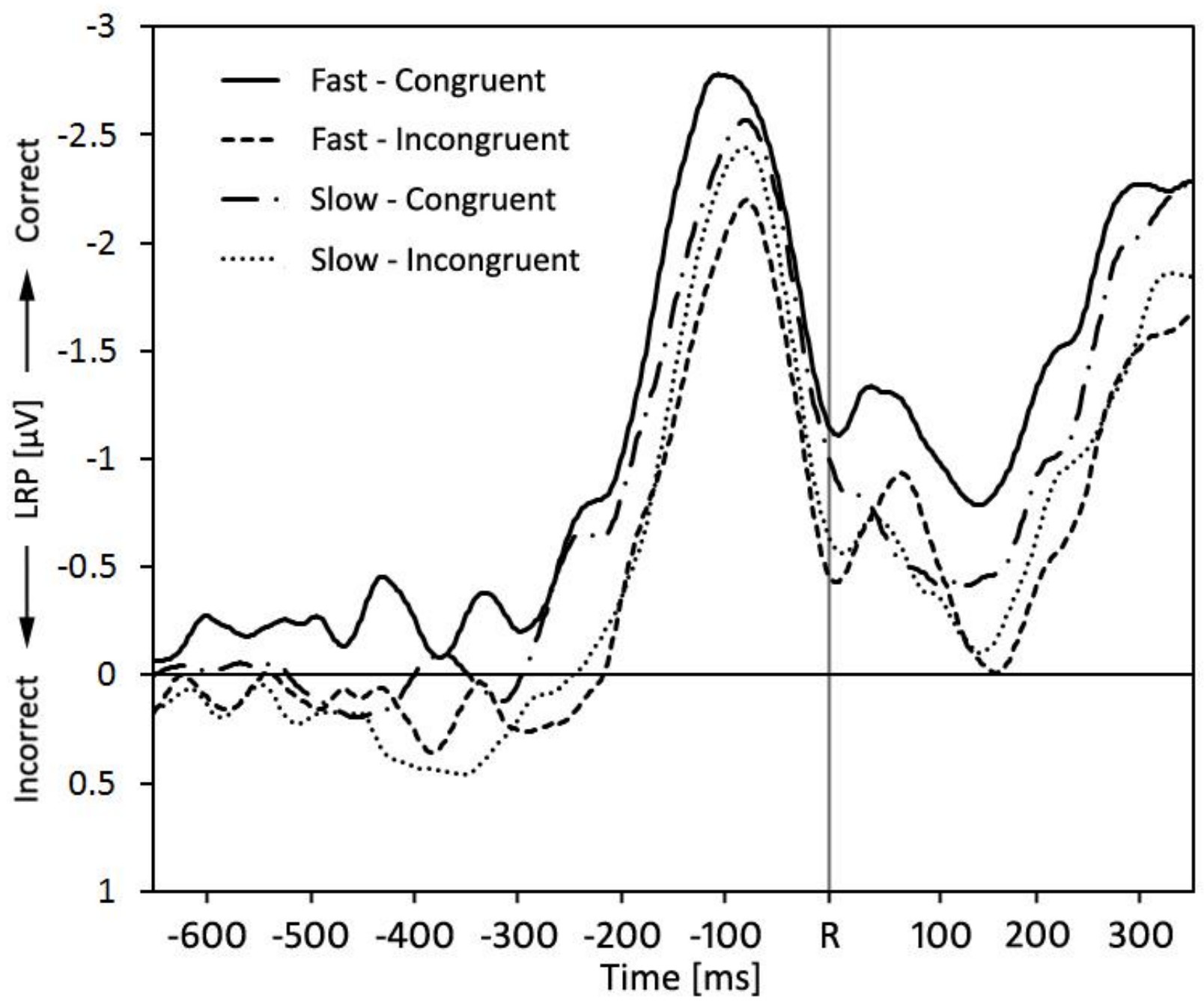


Figure 6

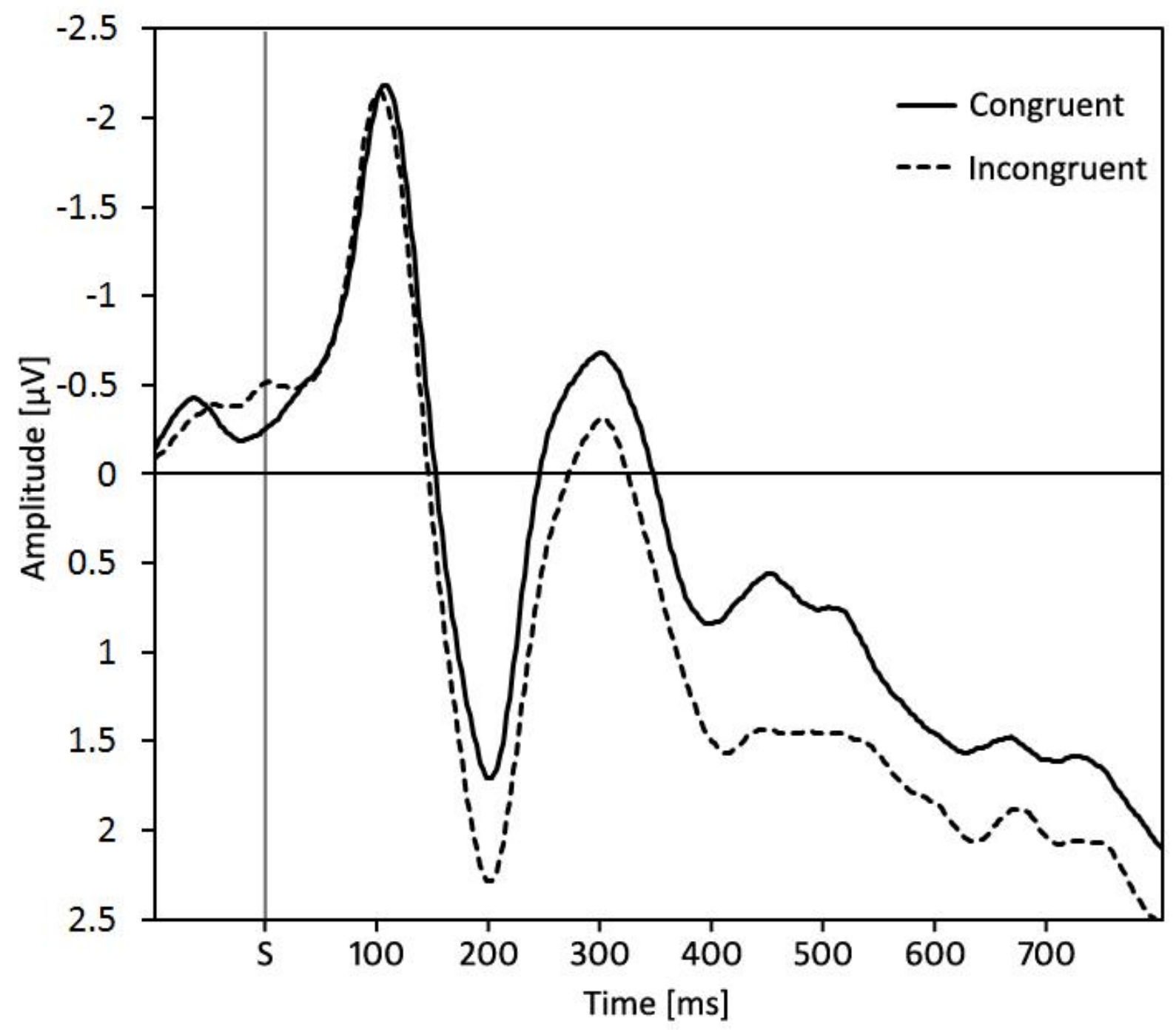


Figure 7

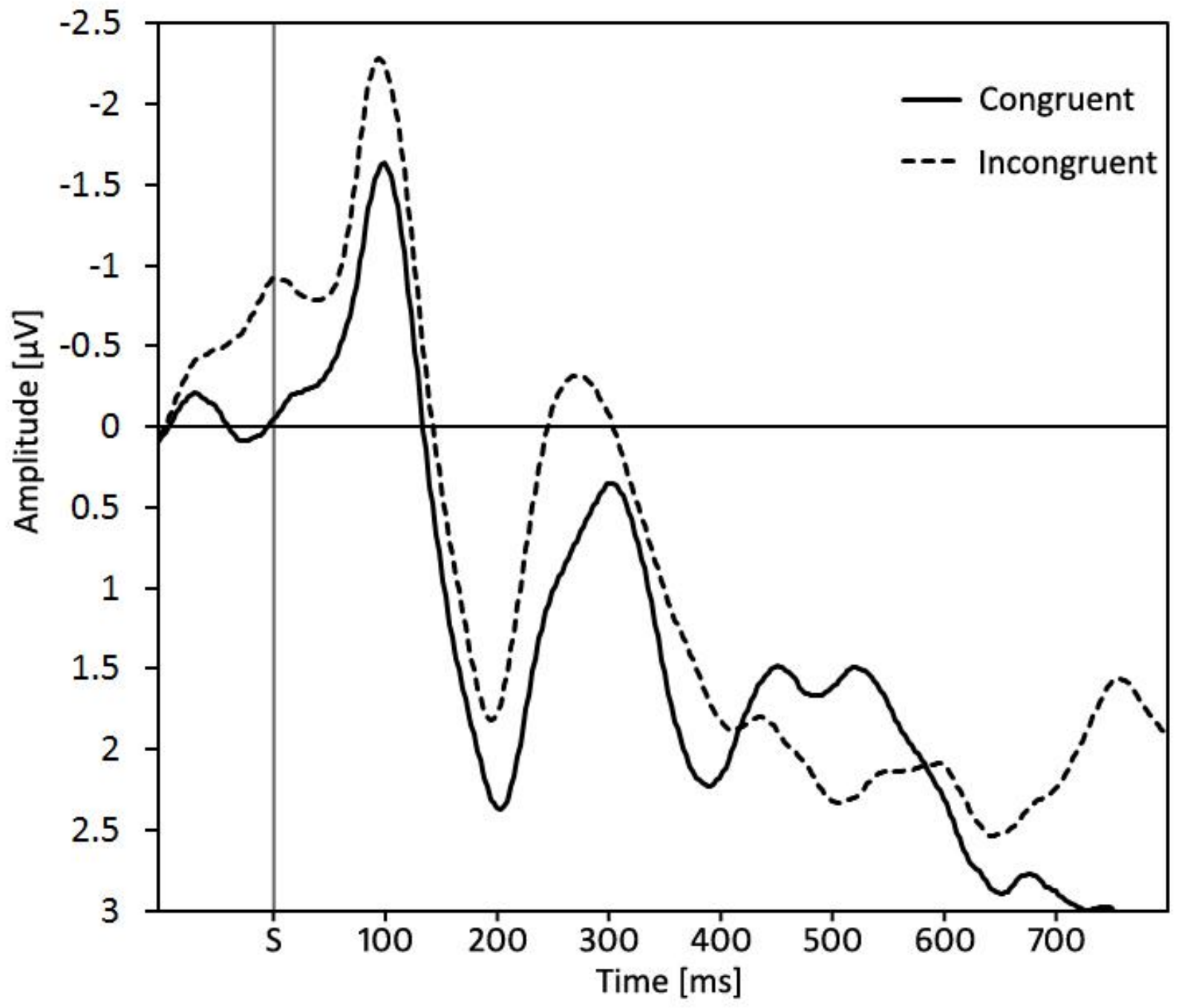

